\title{
Video Article \\ A Lectin HPLC Method to Enrich Selectively-glycosylated Peptides from Complex Biological Samples
}

\author{
Eric Johansen ${ }^{1}$, Birgit Schilling ${ }^{2}$, Michael Lerch ${ }^{1}$, Richard K. Niles ${ }^{1}$, Haichuan Liu ${ }^{1}$, Bensheng Li ${ }^{2}$, Simon Allen ${ }^{1}$, Steven C. Hall ${ }^{1}$, H. \\ Ewa Witkowska ${ }^{1}$, Fred E. Regnier ${ }^{3}$, Bradford W. Gibson ${ }^{2}$, Susan J. Fisher ${ }^{1}$, Penelope M. Drake ${ }^{1}$ \\ ${ }^{1}$ Obstetrics, Gynecology and Reproductive Sciences, University of California, San Francisco - UCSF \\ ${ }^{2}$ Buck Institute for Age Research \\ ${ }^{3}$ Department of Chemistry, Purdue University
}

Correspondence to: Bradford W. Gibson at bgibson@buckinstitute.org

URL: https://www.jove.com/video/1398

DOI: doi:10.3791/1398

Keywords: Basic Protocols, Issue 32, Lectins, chromatography, glycopeptides, glycoproteins, biomarker discovery

Date Published: 10/1/2009

Citation: Johansen, E., Schilling, B., Lerch, M., Niles, R.K., Liu, H., Li, B., Allen, S., Hall, S.C., Witkowska, H.E., Regnier, F.E., Gibson, B.W., Fisher, S.J., Drake, P.M. A Lectin HPLC Method to Enrich Selectively-glycosylated Peptides from Complex Biological Samples. J. Vis. Exp. (32), e1398, doi:10.3791/1398 (2009).

\section{Abstract}

Glycans are an important class of post-translational modifications. Typically found on secreted and extracellular molecules, glycan structures signal the internal status of the cell. Glycans on tumor cells tend to have abundant sialic acid and fucose moieties. We propose that these cancerassociated glycan variants be exploited for biomarker development aimed at diagnosing early-stage disease. Accordingly, we developed a mass spectrometry-based workflow that incorporates chromatography on affinity matrices formed from lectins, proteins that bind specific glycan structures. The lectins Sambucus nigra (SNA) and Aleuria aurantia (AAL), which bind sialic acid and fucose, respectively, were covalently coupled to POROS beads (Applied Biosystems) and packed into PEEK columns for high pressure liquid chromatography (HPLC). Briefly, plasma was depleted of the fourteen most abundant proteins using a multiple affinity removal system (MARS-14; Agilent). Depleted plasma was trypsindigested and separated into flow-through and bound fractions by SNA or AAL HPLC. The fractions were treated with PNGaseF to remove N-linked glycans, and analyzed by LC-MS/MS on a QStar Elite. Data were analyzed using Mascot software. The experimental design included positive controls-fucosylated and sialylated human lactoferrin glycopeptides-and negative controls-high mannose glycopeptides from Saccharomyces cerevisiae - that were used to monitor the specificity of lectin capture. Key features of this workflow include the reproducibility derived from the HPLC format, the positive identification of the captured and PNGaseF-treated glycopeptides from their deamidated Asn-Xxx-Ser/Thr motifs, and quality assessment using glycoprotein standards. Protocol optimization also included determining the appropriate ratio of starting material to column capacity, identifying the most efficient capture and elution buffers, and monitoring the PNGaseF-treatment to ensure full deglycosylation. Future directions include using this workflow to perform mass spectrometry-based discovery experiments on plasma from breast cancer patients and control individuals.

\section{Video Link}

The video component of this article can be found at https://www.jove.com/video/1398/

Protocol

\section{1) Prepare lectin-conjugated POROS columns}

1. Put on a mask to protect against inhalation of POROS-AL beads during steps $1.1-1.2$. Weigh out the desired amount of POROS beads (100 $\mathrm{mg}$ beads $/ 300 \mu \mathrm{l}$ final volume) and transfer into a clean eppendorf tube.

2. Wash beads with addition of $1 \mathrm{~mL}$ phosphate-buffered saline (PBS). Pellet beads by centrifugation in a microcentrifuge at maximum speed for 3 minutes. Remove supernatant and repeat the wash.

3. Weigh out the desired amount of unconjugated lectin (1 - $4 \mathrm{mg} / 200 \mu \mathrm{l}$ beads) and transfer to a clean eppendorf tube. Add PBS to form a 5 $20 \mathrm{mg} / \mathrm{mL}$ solution. Reserve $25 \mu \mathrm{l}$ of this solution.

4. Transfer the remaining lectin solution to the POROS beads. Add sodium cyanoborohydride to a final concentration of $50 \mathrm{mM}$. Place the tube on a rocker and react overnight at room temperature. (Note: sodium cyanoborohydride is toxic and must be handled in a fume hood. Contaminated waste must be disposed of appropriately.)

5. Pellet the POROS beads as in step 1.2. Remove supernatant and save as the post-conjugation solution.

6. Wash beads with $1 \mathrm{~mL}$ Quenching Buffer (1 M Tris-Cl, $\mathrm{pH}$ 7.4). Pellet beads as in step 1.2 and safely discard supernatant.

7. Block the remaining reactive sites on the POROS beads with $1 \mathrm{~mL}$ Quenching Buffer. Add sodium cyanoborohydride to a final concentration of $50 \mathrm{mM}$. Place the tube on a rocker and incubate at room temperature for $30 \mathrm{~min}$.

8. Pellet beads as in step 1.2 and discard supernatant. 
9. Wash beads with $1 \mathrm{~mL} 1 \mathrm{M} \mathrm{NaCl}$. Pellet beads and discard supernatant. Repeat four times for a total of five washes. The beads are now ready to pack.

10. To determine the amount of protein that was conjugated to the POROS beads, perform a protein concentration assay on the pre-conjugated and post-conjugated lectin solutions. The difference in concentrations is the amount of protein that was conjugated to the beads. The amount of protein conjugated per bead volume equals the concentration of lectin on the beads. Target lectin concentrations are between $2-20 \mathrm{mg} /$ $\mathrm{mL}$.

\section{2) Packing the lectin-conjugated POROS beads into a PEEK column}

1. Assemble the packing system (description follows) and support it on a metal ring stand. The packing system consists of, from bottom to top, pressure restrictor, end column coupler 1 , frit, column $(2 \times 50 \mathrm{~mm})$, end column coupler 2 , column connector, end column coupler 3 , column $(4.5 \times 50 \mathrm{~mm})$, end column coupler 4 and end fitting. The upper column serves as a reservoir for the packing material.

2. Resuspend the POROS beads in desired volume of Buffer $\mathrm{A}(10 \mathrm{mM}$ Tris buffer, $\mathrm{pH} 7.4,150 \mathrm{mM}$ sodium chloride, $10 \mathrm{mM}$ calcium chloride, $10 \mathrm{mM}$ magnesium chloride). If packing one column ( 200 $\mu \mathrm{l}$ bed volume), resuspend in $400 \mu \mathrm{l} \mathrm{Buffer} \mathrm{A}$.

3. Transfer conjugated POROS beads into the upper column (reservoir). Add Buffer A to the reservoir until the buffer reaches the top of the column. Gently place the end fitting onto the top of the column, trying to avoid air bubbles in the column. Connect the end fitting of the upper column to the HPLC system.

4. Pack the column by flowing Buffer $A$ through the system. Start with a flow rate of $0.5 \mathrm{~mL} / \mathrm{min}$. Increase the flow rate by $0.5 \mathrm{~mL} / \mathrm{min}$ each minute until either $4 \mathrm{~mL} / \mathrm{min}$ or the maximum pressure $(3000 \mathrm{psi})$ has been reached. Continue at the maximum possible flow rate until at least $35 \mathrm{~mL}$ of Buffer A have passed through the column.

5. Turn off the pumps and allow the pressure on the column to drop to $<20 \mathrm{psi}$.

6. Gently, disassemble the packing system, starting from the top. When the end column coupler 2 is reached, remove carefully. Some packed material should be extruding from the top of the column. With a razor blade or similar sharp edge, gently wipe away the extruding beads, leaving a bead surface that is even with the top of the column. Do not apply pressure to the beads while doing this.

7. Disengage the packed column from the ring stand. Place a new frit into a new end coupler and turn the packed column over to connect this with the frit/end coupler on the open (formerly top) column end.

8. Label the column appropriately. It is now ready for use. When not in use, the column may be stored in PBS with $0.02 \%$ sodium azide at $4{ }^{\circ} \mathrm{C}$ for up to 6 months.

\section{3) Program the HPLC method}

1. The details of programming your HPLC will vary according to the specifics of the manufacturer's software. We use a Michrom Paradigm MG4 HPLC. On this machine, methods are built and accessed under the "Setup" tab at the top of the screen. Program the following method:

\begin{tabular}{|l|l|l|l|}
\hline Time & Flow rate $(\mu \mathrm{l} / \mathrm{min})$ & $\% \mathrm{~A}$ & $\% \mathrm{~B}$ \\
\hline $0: 00$ & 50 & 100 & 0 \\
\hline $9: 00$ & 50 & 100 & 100 \\
\hline $9: 01$ & 500 & 0 & 100 \\
\hline $13: 50$ & 500 & 0 & 0 \\
\hline $13: 51$ & 3000 & 100 & 0 \\
\hline $19: 50$ & 3000 & 100 & 0 \\
\hline
\end{tabular}

Buffer A: $10 \mathrm{mM}$ Tris buffer, $\mathrm{pH}$ 7.4, $150 \mathrm{mM}$ sodium chloride, $10 \mathrm{mM}$ calcium chloride, $10 \mathrm{mM}$ magnesium chloride

Buffer B: $0.5 \mathrm{M}$ acetic acid

The UV detector should be programmed to read at $280 \mathrm{~nm}$ from 0:00 to 19:50. The y-axis should be adjusted to detect low-absorbance levels, e.g., 0 - $50 \mathrm{AU}$.

2. If an autosampler is available, program the following schedule for fraction collection. Otherwise, collect the fractions by hand as indicated when performing chromatography.

\begin{tabular}{|l|l|l|}
\hline Fraction & $\begin{array}{l}\text { Delay to collection from sample } \\
\text { injection }\end{array}$ & Duration of collection (min) \\
\hline Flow-through & 2.8 & 4.1 \\
\hline Bound & 9 & 2.6 \\
\hline
\end{tabular}

\section{4) Prepare samples for HPLC}

1. Prior to use in this protocol, human plasma should be depleted of the 14 most-abundant proteins using a MARS column (Agilent; Santa Clara, CA). Depleted plasma, human lactoferrin and yeast invertase should be individually trypsin-digested and desalted as previously described ${ }^{1}$.

2. To prepare the lectin-specific lactoferrin standard, perform chromatography on 50 ug trypsin-digested lactoferrin over either the AAL or the SNA column using the method in Part 3 . The bound fraction will contain lectin-specific lactoferrin glycopeptides. Neutralize the samples as in Part 4.4, and desalt as in Part 6. Resuspend the sample in $1 \mathrm{~mL}$ Buffer A. The resulting sample is ready for use.

3. Three experimental replicates will be performed. To prepare sample for three replicates, add 3 pmol of trypsin-digested invertase and 1 $\mu \mathrm{l}$ of lectin-specific lactoferrin (either AAL-LAC or SNA-LAC) to $30 \mu \mathrm{l}$ MARS-depleted, trypsin-digested, plasma equivalents (PE). A PE is 
defined as the volume of non-processed/intact/original plasma from which a given amount of processed plasma (MARS-depleted and trypsin digested) was derived. Add Buffer A to the sample to reach a final volume of $330 \mu$ l.

4. Add neutralization buffer (1 M Tris $\mathrm{pH} 8.0)$, to the sample vials that will collect eluate. The number and volume of vials you work with will depend upon the constraints of your autosampler. For the Paradigm MG4, one vial collects the flow-through and two collect the eluate. It will require approximately $1.5 \mathrm{~mL}$ Tris buffer to neutralize $1 \mathrm{~mL}$ of eluate. Target neutralized $\mathrm{pH}$ is $7.0-8.0$; test test final $\mathrm{pH}$ with $\mathrm{pH}$ indicator paper.

\section{5) Perform chromatography}

1. All the blank and sample runs that follow will use the method described in Part 1. The column and buffers are at room temperature while in use. Columns are stored at $4^{\circ} \mathrm{C}$, while buffers are stored at room temperature.

2. Attach either the AAL or the SNA lectin column to the HPLC system and run two blank methods (injecting only Buffer A). Ensure that the lectin column corresponds to the lectin-specific lactoferrin (Part 2).

3. Chromatograph the three plasma samples, injecting a blank in between each injected sample, for a total of $7 \mathrm{HPLC}$ runs. Fractions from blank runs need not be collected.

\section{6) Desalt collected fractions}

For each fraction use one $1 \mathrm{~mL}$ Waters Oasis HLB SPE cartridge as follows:

1. Attach the number of required cartridges to vacuum manifold.

2. Wet each cartridge with $1 \mathrm{~mL} 80 \% \mathrm{ACN}$ in $1 \%$ formic acid (vacuum gauge on manifold should read $5-20 \mathrm{lnHg}$ ).

3. Equilibrate cartridges with $1.5 \mathrm{~mL} 0.1 \%$ formic acid (vacuum gauge on manifold should read $5-20 \mathrm{InHg}$ )

4. Load entire volume of one sample onto one cartridge (Vacuum gauge on manifold should read $2-2.5 \mathrm{InHg}$, and flow rate should not exceed 1 $\mathrm{mL} / \mathrm{min}$.)

5. Wash cartridges with $3 \times 1 \mathrm{~mL} 0.1 \%$ formic acid (Vacuum gauge on manifold should read $5-20 \mathrm{lnHg}$ )

6. Elute peptides/glycopeptides into labeled eppendorf tubes with $1 \times 1 \mathrm{~mL} 80 \%$ acetonitrile in $0.1 \%$ formic acid. (Vacuum gauge on manifold should read $2-2.5 \mathrm{lnHg}$, and flow rate should not exceed $1 \mathrm{~mL} / \mathrm{min}$.) A single collection tube should be used for each cartridge.

7. Neutralize eluate by adding $60 \mu \mathrm{l} 0.5 \mathrm{M}$ ammonium bicarbonate to each collection tube. Target neutralized $\mathrm{pH}$ is is $7.0-8.0$; test final pH with $\mathrm{pH}$ indicator paper.

8. Concentrate eluted peptides/glycopeptides to $\sim 50 \mu \mathrm{L}$ by running them on a vacuum centrifuge (e.g., a Thermo Savant SpeedVac) for $\sim 2$ hrs @ $35^{\circ} \mathrm{C}$.

\section{7) PNGase F-digestion}

1. Test sample $\mathrm{pH}$ to ensure it is between $7.0-8.0$. If needed, add $50 \mathrm{mM}$ ammonium bicarbonate to increase $\mathrm{pH}$. If final sample volume is > $100 \mu \mathrm{l}$, speedvac sample until it is between $50-100 \mu \mathrm{l}$.

2. Add $0.5 \mu \mathrm{l}(250 \mathrm{U})$ glycerol-free $P N G a s e ~ F$ to each sample tube and incubate at $37^{\circ} \mathrm{C}$ overnight.

\section{8) Zip Tip samples}

1. Starting with PNGase F-digested samples, ziptip each one as follows.

2. Wet ziptip with $10 \mu \mathrm{l} 100 \%$ acetonitrile, followed by $10 \mu \mathrm{l} 80 \%$ acetonitrile, $0.1 \%$ formic acid.

3. Equilibrate ziptip with $20 \mu \mathrm{l} 0.1 \%$ formic acid.

4. Load the sample onto the ziptip by pipetting it up and down through the ziptip matrix 10 times.

5. Wash the ziptip by pipetting $10 \mu \mathrm{l} 0.1 \%$ formic acid, 5 times.

6. Elute the sample into a clean eppendorf tube with $10 \mu \mathrm{l} 80 \%$ acetonitrile, $0.1 \%$ formic acid.

7. Repeat the elution and add the second $10 \mu$ into the same tube.

8. Speedvac the samples to a volume of $<2 \mu \mathrm{l}$.

9. Resuspend samples in $20 \mu \mathrm{l} 0.1 \%$ formic acid. Samples are now ready for analysis by LC-MS/MS.

\section{9) Representative Results:}

The POROS conjugation typically yields on-bead lectin concentrations in the range of $2-20 \mathrm{mg} / \mathrm{mL}$. This is optimal for affinity chromatography.

Lectin chromatography of trypsin-digested MARS-depleted human plasma typically enriches glycopeptides in the bound fraction. As the glycopeptides are PNGase F-treated prior to LC-MS/MS, they are identified as peptides with the N-linked consensus sequence, NXS/T (where $\mathrm{X}$ is any residue but proline), in which the asparagine has been converted to an aspartic acid through the action of PNGase F. Peptides with these characteristics are considered to be deglycosylated peptides (or "deglycopeptides"). On average the flow-through (FT) fraction from AAL or SNA chromatography contains $2-4 \%$ deglycopeptides, whereas $30-50 \%$ of the peptides recovered in the bound fraction are deglycopeptides. Typically, using a QStar Elite, 1000-1300 peptides will be identified in the FT fraction, and 200-400 peptides will be identified in the bound fraction. Two or more distinct Invertase deglycopeptides should be observed in the FT fraction and two or more distinct lactoferrin deglycopeptides in the bound fraction (Table 1). 


\begin{tabular}{|c|c|c|}
\hline Protein ID & Peptide sequence $^{*}$ & Theoretical m/z $^{-1}$ \\
\hline INVERTASE (P10594) & K.NPVLAANSTQFR.D & 1657.85 \\
INVERTASE (P00724) & K.AEPILNISNAGPWSR.F & 1624.79 \\
\hline LACTOFERRIN (P02788) & R.NGSDCPDKFCLFQSETK.N & 2031.87 \\
LACTOFERRIN (P02788) & R.PFLNWTGPPEPIEAAVAR.F & 1964.94 \\
LACTOFERRIN (P02788) & R.TAGWNIPMGLLFNQTGSCK.F & 2094 \\
LACTOFERRIN (P02788) & R.TAGWNVPIGTLRPFLNWTGPPEPIEAAVAR.F & 3229.7 \\
\hline
\end{tabular}

Table 1.

\section{Discussion}

This protocol provides a quick method for isolating and identifying glycopeptides in a glycan-specific manner. As glycosylation varies extensively within an organism, particularly during development and pathogenesis, this technique may be applicable to address a wide variety of questions. In particular, we are using the method to selectively enrich glycopeptides that have been modified with cancer-associated epitopes as a first step towards biomarker discovery.

\section{Acknowledgements}

This work was supported by the Clinical Proteomic Technologies for Cancer initiative, 5U24CA126477-04.

\section{References}

1. Keshishian, H. et al. Quantitative, multiplexed assays for low abundance proteins in plasma by targeted mass spectrometry and stable isotope dilution. Mol Cell Proteomics 6, 2212-2229 (2007). 